\title{
Determination of Sintering Parameters from In-situ Aberration-Corrected STEM Imaging of Coalescence in Pt Nanoparticles
}

\author{
M.A. Asoro, ${ }^{*}$ D. Kovar, ${ }^{*}$ Y. Shao-Horn, ${ }^{* *}$ L.F. Allard*** and P.J. Ferreira*
}

*Materials Science and Engineering Prog., University of Texas at Austin, Austin, TX, 78712, USA

**Dept. of Mechanical Engr, Massachusetts Institute of Technology, Cambridge, MA, 02139, USA

***Materials Science and Technology Div., Oak Ridge National Laboratory Oak Ridge, TN, 37831

Nanoparticles are currently of great scientific interest due to their large number of possible applications - such as catalysts in fuel cells and as delivery vehicles for medicine. However, during processing or usage, nanoparticles have a strong tendency to agglomerate and coalesce over short time scales, even at room temperature, which can lead to significant changes in behavior and performance. Although a reduction in sintering temperature is predicted from simple scaling models such as Herring's law, it is important to know the relevant diffusivities to predict sintering kinetics of nanoparticles [1-3].

In the present work, we have used an aberration-corrected JEOL 2200FS scanning transmission electron microscope (STEM), equipped with a high-angle annular dark-field (HA-ADF) detector to monitor the sintering process of Pt nanoparticles with $2.8 \mathrm{~nm}$ nominal size. This experiment provides real-time dynamic information for a direct investigation of the evolution of sintering, which post-mortem TEM observations are not capable of conveying.

A sequence of aberration-corrected HA-ADF STEM images showing the coalescence of Pt nanoparticles under the influence of the electron beam at ambient temperature is shown in Fig. 1. To minimize the influence of the electron beam on the sintering process, the beam was blanked after each image was taken. The particle radius, neck radius, particle-to-particle distance and dihedral angle were measured from the HA-ADF STEM images (Fig. 2), using Gatan Digital Micrograph software. The measured values are shown in Table 1 . These values can subsequently be used to calculate fundamental variables, such as surface and grain boundary diffusion coefficients, as well as grain boundary mobility. For example, if we consider surface diffusion to be dominant, the surface diffusion coefficient $D_{\mathrm{s}}$ is calculated to be within the range $3.47 \times 10^{-20} \mathrm{~cm}^{2} / \mathrm{s}-1.19 \times 10^{-19} \mathrm{~cm}^{2} / \mathrm{s}$ for Pt nanoparticles, which agrees well with the calculated value of $D_{s}$ for bulk $P t\left(1.13 \times 10^{-19} \mathrm{~cm}^{2} / \mathrm{s}\right)$ [4]. However, as the calculations are based on a single experiment (Fig. 1), we should take these results with caution. Nevertheless, the in-situ aberration-corrected HA-ADF STEM proves to be a valuable technique to study the coalescence of very small nanoparticles, particularly those which are supported on a carbon substrate, such as the case of Pt catalysts for fuel-cell applications [5].

\section{References}

[1] M. Yeadon et al, Nanostructured Materials 10 (1998) 731-739.

[2] Y. Champion et al, Materials Science and Engineering 360 (2003) 258-263.

[3] J. R. Groza, Nanostructured Materials 12 (1999) 987-992.

[4] M. L. Marcos, J. González Velasco, Chemical Physics Letters 283 (1998) 391-394.

[5] This research at the Oak Ridge National Laboratory's High Temperature Materials Laboratory was sponsored by the U. S. Department of Energy, Office of Energy Efficiency and Renewable Energy, Vehicle Technologies Program. 


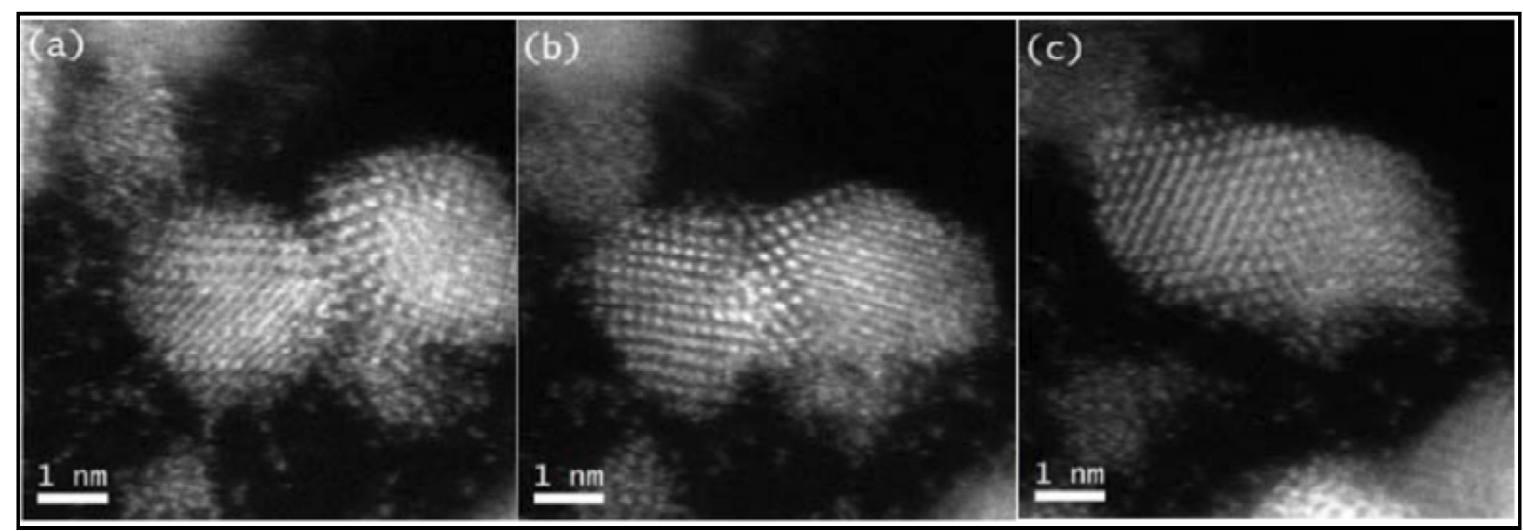

Fig. 1: A sequence of STEM HA-ADF images showing the coalescence of Pt nanoparticles. The images, left to right. were recorded at intervals of 20 seconds.
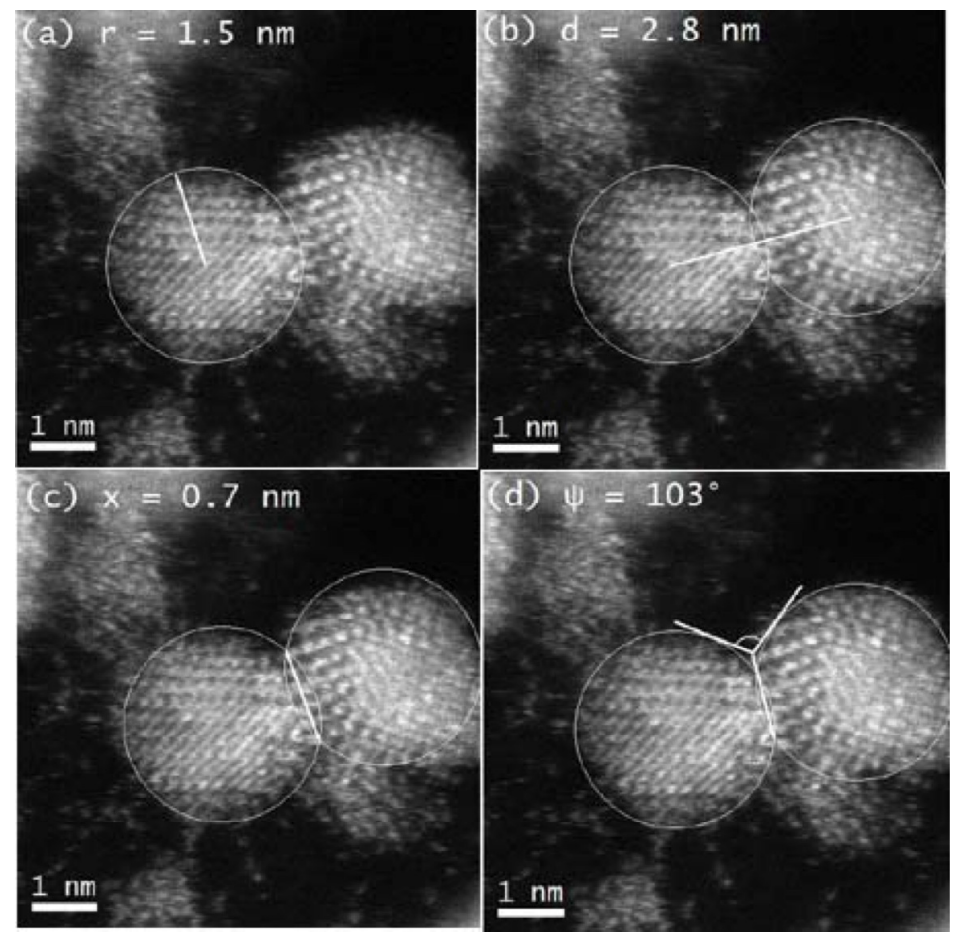

Fig. 2: Initial HA-ADF image showing the measurement geometry for (a) particle radius (b) particleto-particle distance (c) neck radius and (d) dihedral angle.

Table 1: Measurements of particle radius, neck radius, particle-to-particle distance and dihedral angle as function of sintering time.

\begin{tabular}{|c|c|c|}
\hline Sintering time (sec) & 15 & 50 \\
\hline Particle radius (nm) & 1.5 & 1.4 \\
\hline Neck radius (nm) & 0.7 & 0.9 \\
\hline Particle-to-particle distance (nm) & 2.8 & 2.7 \\
\hline x/a & 0.467 & 0.643 \\
\hline Dihedral angle & 103 & 155 \\
\hline
\end{tabular}

